\title{
连续性肾脏代替治疗应用于ICU重症急性肾功能衰竭中 的价值观察
}

\author{
王 兰* 李慧明 任晓玲 \\ 国药葛洲坝中心医院，湖北 443000
}

摘 要: 目的: 研究连续性肾脏替代治疗在ICU重症急性肾功能衰竭中的应用和护理对策。方法: 选取2019年1 月1日 2020年12月1日我院在ICU重症室进行治疗的急性肾功能衰竭患者50例作为研究对象, 进行总结分析。结果: 给予急性肾功能衰竭连续性肾脏替代治疗和制定护理对策护理下, 11例患者好转出院, 21 例患者治愈, 抢救成功率为 $88.89 \%$, 治疗后患者的心理、平均血压以及肌酐等值均得到了一定的控制。结论：对实施连续性肾脏替代治疗的ICU 重症急性肾功能衰竭患者实施综合护理千预对策有利于提高抢救成功率，降低并发症的发生概率，值得推广使用。

关键词：急性肾功能衰竭; 连续性肾脏治疗; 护理对策

\section{一、引言}

重症急性肾衰竭属于常见的一种疾病, 该疾病一般是由于严重感染、严重创伤或是颅脑损伤引起的 ${ }^{[1]}$ 。针对该疾 病的治疗, 临床中一般采用血液净化疗法, 以有效清除血液中的有毒有害物质及自由基, 减轻肾脏负担, 但是血液净 化治疗不良反应较多, 患者耐受力差 ${ }^{[2]}$ 。近几年, 连续肾脏替代疗法逐步在临床中推广, 并取得了比较理想的治疗效 果。实践结果表明 ${ }^{[3]}$, 连续肾脏替代疗法可以有效减少重症急性肾衰竭患者的病死率, 这主要是由于续肾脏替代疗法 具有较高的溶质清除率，并且生物相性好，血流动力学稳定。

\section{二、资料与方法}

(一) 一般资料

研究对象选取2019年1月1日～2020年12月1日我院收治的50例ICU重症急性肾功能衰竭患者, 按照随机数字法, 将患 者分为对照组和观察组, 各25例。其中对照组患者男12例、女13例, 观察组男17例、女8例; 两组患者年龄 $25 \sim 78$ 岁, 观察组和对照组患者平均年龄分别为 $(61.15 \pm 10.11)$ 岁、 $(62.38 \pm 12.39)$ 岁。两组患者的性别、年龄等临床资料无明 显差异, 差异无统计学意义 $(P>0.05)$ 。本研究经医院医学伦理委员会审核批准, 患者及其家属均对本研究知情同意。

\section{(二) 治疗方法}

研究组使用连续性肾脏替代治疗, 设备使用费森精神尤斯连续血液净化系统和配套的血滤器, 实施股静脉或是颈内 静脉置管方式, 选择连续性静脉一静脉血液过滤模式 ${ }^{[4]}$ 。依据实际情况调节水电解质酸碱浓度, 将稀释后的碳酸氢盐作 为置换液, 用量为 $3 \mathrm{~L} / \mathrm{h}$, 治疗期间使用低分子肝素行抗凝治疗, 每次治疗 $6 \sim 10 \mathrm{~h}$ 左右, 病情严重的患者进行 $24 \mathrm{~h}$ 不间断 治疗，每隔半小时测量患者血压、心率等体征指标。若患者血压异常，则缩短测量间隔时间，实施持续监测 ${ }^{[5]}$ 。

对照组患者实施间歇性血液净化治疗, 使用碳酸氢盐作为透析液 ${ }^{[6]}$, 实施股静脉或是颈内静脉置管, 设备使用F60 聚砜膜透析器, 面积 $1.3 \mathrm{~m}^{2}$, 透析量设置为 $200 \sim 350 \mathrm{~mL} / \mathrm{min}$, 透析液流量速度设置为 $500 \mathrm{~mL} / \mathrm{min}$, 隔天透析, 4 小时/ 次，治疗过程中注意观察患者体征和病情。

\section{(三) 观察指标}

1. 比较两组患者的血液生化指标的变化情况。

2. 比较两组患者的存活率及不良反应发生率。

(四) 统计学方法

用SPSS2 10 专业统计学软件对本次研究涉及的计量资料和计数资料进行数据处理, 计量资料与计数资料分别以均 数 \pm 标准差和 $[n(\%)]$ 表示, 检验方法分别采取 $t$ 检验和 $Z$ 检验, 均以 $P<0.05$ 表示差异具有统计学意义。

*通讯作者: 王兰, 1988年12月, 女, 汉, 湖北丹江口人, 就职于国药葛洲坝中心医院, 主管护师, 本科。研究 方向: 护理。 


\section{三、结果}

（一）比较两组患者的血液生化指标的变化情况

比较两组患者的血液生化指标的变化情况治疗后, 两组患者的血清中BUN、Cr、 $\mathrm{K}^{+}$水平较治疗前均有明显降低, 但观察组的上述血液生化指标水平均明显低于对照组，差异有统计学意义 $(P<0.05)$, 详情见表1。

\section{表1 比较两组患者的血液生化指标的变化情况 $(\bar{\chi} \pm S)$}

\begin{tabular}{ccccccc}
\hline \multirow{2}{*}{ 组别 } & \multicolumn{2}{c}{$\mathrm{BUN}(\mathrm{mg} / \mathrm{dL})$} & \multicolumn{2}{c}{$\mathrm{Cr}(\mu \mathrm{mol} / \mathrm{L})$} & \multicolumn{2}{c}{$\mathrm{K}^{+}(\mu \mathrm{mol} / \mathrm{L})$} \\
& 治疗前 & 治疗后 & 治疗前 & 治疗后 & 治疗前 & 治疗后 \\
\hline 对照组 $(n=25)$ & $43.1 \pm 9.0$ & $25.6 \pm 6.28$ & $356.5 \pm 127.38$ & $238.6 \pm 85.35$ & $5.7 \pm 2.09$ & $4.5 \pm 1.18$ \\
观察组 $(n=25)$ & $42.6 \pm 8.7$ & $21.1 \pm 5.19$ & $49.3 \pm 124.49$ & $221.1 \pm 78.52$ & $5.7 \pm 2.02$ & $3.7 \pm 14.02$ \\
$P$ & $>0.05$ & 0.01 & $>0.05$ & 0.00 & $>0.05$ & 0.00 \\
$t$ & 1.28 & 3.01 & 2.34 & 1.84 & 2.12 & 2.45 \\
\hline
\end{tabular}

(二) 比较两组患者不良反应发生率

比较两组患者的生存率及不良反应发生率经治疗后, 观察组患者共计有 21 人存活, 生存率为 $84.0 \%$, 对照组共计 有 22 人存活, 生存率为 $88.0 \%$, 组间比较, 两组患者生存率无明显差异, 差异无统计学意义 $(P>0.05)$, 在不良反应 发生率上, 两组患者也无明显差异, 差异无统计学意义 $(P>0.05)$, 具体不良反应发生情况如表2所示。

表2 两组患者不良反应发生率比较 $[\boldsymbol{n}(\%)]$

\begin{tabular}{cccccc}
\hline 组别 & 穿刺出血 & 电解质紊乱 & 心律失常 & 低血压 & 总有效率 $(\%)$ \\
\hline 对照组 $(n=41)$ & 2 & 1 & 1 & 1 & 1 \\
观察组 $(n=41)$ & 2 & 0 & & \\
$P$ & - & 1.21 & & \\
$\chi^{2}$ & - & 3.35 & & \\
\hline
\end{tabular}

\section{四、讨论}

急性肾功能衰竭 ( ARF ) 是患者受多种因素共同影响发生的一种急性肾损害, 属临床危重症, 据相关统计数据显 示, 约有 $20 \%$ 患者会出现急性尿毒症、高血钾综合征等严重症状, 且整体病死率高达 $10 \%$ 以上。相较于普通患者, ICU重症ARF患者病情更为严重及复杂, 常合并有其他脏器损伤, 血液动力学异常等不良状况, 给治疗带来了较大难 度[7]。目前临床对其主要采取血液净化治疗，应用较为广泛的手段有间歇性血液透析治疗。

连续性肾脏替代疗法是继药物、手术治疗后的第三种疗法, 也是一种治疗彻底的方法。该疗法作为临床新型的血 液净化技术, 相较于传统的血液透析具备操作简便、血流动力学稳定、溶质的清除率高等优点, 有效提高了 ICU重症 急性肾功能衰竭患者的生活质量 ${ }^{[8]}$ 。现临床给予连续性肾脏替代治疗在ICU重症急性肾衰竭中的患者一定的护理干预 对策，不仅提高了整体的技术水平，也为抢救患者而争取了更多的时间。

间歇性血液透析治疗是临床中治疗肾功能衰竭的常用方式, 其主要是通过体外净化系统清除血液中的垃圾和毒素 物质, 从而确保患者体内循环稳定, 保障各个器官的运行, 避免发生更为严重的脏器功能衰竭情况。连续性肾脏替代 治疗在临床中使用也比较普遍，与间歇性的血液透析治疗相比，该疗法可以有效控制患者病情，降低了患者的死亡。 连续性肾脏替代治疗可以保证患者血流动力学的稳定, 而间歇性血液净化治疗无法做到这一点, 其原因在于间歇性血. 液净化治疗下, 患者体液容量变化快、血气参数变化快, 而且存在血膜反应, 代谢分解率较高, 从而导致患者躯体不 耐受。而连续性肾脏替代治疗实现了缓慢且等渗性脱水, 并且可以灵活地调节水和溶质清除参数, 从而确保了血流动 力学的稳定, 所发生的不良反应也比较少, 患者耐受性较好。

本次研究表明, 给予急性肾功能衰竭患者连续性肾脏替代治疗和护理干预, 36名患者中有11名患者病情明显好转 甚至顺利出院, 21 例患者完全治愈, 较传统的治疗, 抢救成功率明显提高; 且患者的心率、肌酐以及尿素氮等值均显 示正常。

\section{五、结语}

综上所述, 对实施连续性肾脏替代治疗的ICU重症急性肾功能衰竭患者实施综合护理干预对策有利于提高抢救成 功率, 降低并发症的发生概率, 值得推广使用。 


\section{参考文献：}

[1]何芳,陆晓翠.ICU重症急性肾功能衰竭中连续性肾脏替代治疗的护理对策分析[J].中外医疗, 2017,36(6):165-167.

[2]杨月香,肖合存,王会.连续性肾拄替代治疗心脏外科术后急性肾功能衰竭26例效果观察及护理 [J]. 齐鲁护理杂志, 2015,21(20):73-74.

[3] 张华丽.连续性血液净化技术救治重症急性肾功能衰竭的护理对策探讨 [ J]. 临床医药文献杂志(电子版), 2016,3(33):6617-6617.

[4]孙玲.急性肾后性肾功能衰竭的术后观察及护理分析[J].国际医药卫生导报, 2015,21(8):1152-1154.

[5]刘旗婷.对接受连续性肾脏替代治疗的重症急性肾功能衰竭患者进行有针对性护理的效果探析 [J]. 当代医药论丛, 2017,15(1):180-181.

[6] 魏瑞.针对性护理在急性肾功能衰竭患者连续性血液净化治疗中的应用分析[J].河南医学研究, 2015,24(8):158-158.

[7]张海霞,刘冰.连续性肾脏替代治疗与间歇性血液透析治疗重症急性肾功能衰竭的效果分析 $[\mathrm{J}]$. 中国实用医药. 2018(26)

[8]吕宝林.连续性血液净化治疗ICU重症急性肾功能衰竭的临床探讨[J].中西医结合心血管病电子杂志. 2019(26). 